\title{
Thomas Diette
}

Université de Lille

ALITHILA

L'Opium du ciel de Jean-Noël Orengo : la quête spirituelle d'un drone, entre ascèse des plaisirs charnels et exhibition des corps

$\mathrm{L}$ e récit sapiosexuel trouve son origine mythique dans la Genèse. Dans le jardin d'Éden, Dieu fait pousser toutes sortes d'arbres, mais parmi ceux-là, deux sont distincts : l'arbre de vie et l'arbre de la connaissance du bien et du mal' ${ }^{1}$. Seul ce dernier est tenu par un interdit: I'homme et la femme ne doivent pas manger les fruits savoureux sous peine de mourir. Mais la suite est connue : poussée par la ruse du serpent, Eve est tentée d'accéder à l'intelligence et mange le fruit défendu. Séduction et savoir se trouvent associés prouvant par-là que l'histoire du désir se mêle à la quête du savoir, ou plutôt de la sagesse².

Le premier acte pour accéder à l'entendement est ainsi un acte de transgression, qui s'est conclu par un châtiment divin qui a engendré la corruption dans leurs vies et dans le monde mais également par la prise en compte de la nudité des deux habitants du paradis. En définitive, la volonté initiale d'accéder au savoir se conclut par le dévoilement des corps.

Dans L'Opium du cie/3, Jean-Noël explore de manière inattendue ces questions ontologiques, en plaçant au centre

\footnotetext{
${ }^{1} \mathrm{Gn} 2$, 9. Si l'arbre de vie se retrouve dans d'autres mythes en Orient, en particulier dans l'épopée de Gilgamesh, l'arbre de la connaissance du bien et du mal est unique et demeure difficile à expliquer.

${ }^{2}$ C. De Mulder, Libido sciendi ; le savant, le désir, la femme, Paris, Seuil, 2012.

3 J.-N. Orengo, L'Opium du ciel, Paris, Grasset, 2017. Pour faciliter la lecture pour la suite de l'article, l'œuvre sera abrégée sous la forme $O C$.
} 
de son roman un drone pourvu d'une conscience. Narrateur certes omniscient mais empêché, exclu du monde par sa nature technicisée, il n'en demeure pas moins un inquisiteur préoccupé par l'essence du monde. À la recherche d'un idéal dans un monde spleenétique, le héros du récit explore les frontières terrestres et célestes en même temps qu'il épie les amours charnelles des humains. Ces plaisirs interdits n'empêchent pas pour autant une quête spirituelle. Doté d'un code de vie, insufflé par ses créateurs, le drone aspire à des plaisirs supérieurs. L'Opium du ciel est le récit d'une nouvelle histoire du désir.

Le drone est une créature hybride à plus d'un titre : entité technologique se nourrissant d'humanité, il oscille de façon permanente entre le vouloir et le pouvoir. Cette double nature lui confère le statut d'un marginal qui doit surpasser sa solitude pour trouver sa voix ${ }^{4}$. Cette quête d'un ascète du plaisir charnel est-elle nécessairement vouée à l'échec ? Dans un monde en déclin, la conquête des sphères célestes est une issue envisagée pour faire advenir une nouvelle forme de savoir. Le drone est une vigie du monde, une manifestation du divin, " présent partout et visible nulle part $\aleph^{5}$, qui se nourrit sans cesse de ses observations. Cette forme moderne d'innutrition a pour ambition le surgissement ou plutôt le dévoilement de la transcendance. Le récit propose une révélation finale, une Apocalypse $^{6}$, qui éclaire une idée nouvelle : l'existence d'une déesse créatrice à l'origine du monde.

${ }^{4}$ En musique, le drone désigne un genre minimaliste faisant usage de bourdons (drones en anglais) et créant un son continu. Dans l'ouverture et la clausule du récit, le drone exprime sa volonté de trouver " la musique facile qui dure longtemps " (Ibidem, p. 9, p. 251).

${ }^{5}$ Référence à une célèbre phrase de Flaubert adressée à Louise Colet dans une lettre en date du 9 décembre 1852, [dans :] Idem, Correspondance, vol. II (juillet 1851 - décembre 1858), J. Bruneau (éd. critique), Paris, Gallimard, 1980, p. 204. Il compare à cette occasion la position de l'auteur et celle de Dieu. De façon analogue, le drone est à la fois figure divine et figure de l'auteur omniscient.

${ }^{6}$ Le dernier livre du Nouveau Testament, également appelé Révélation de Jésus-Christ, dévoile à l'évangéliste Jean le sens divin de son époque. Dans 


\section{Désir et frustration}

Le premier chapitre du roman, " L'après-midi d'un drone ${ }^{7}$, rend compte de l'incapacité physique du drone à agir sur le monde. II est privé du pouvoir de changer ce qui touche au terrestre. Son élément est le ciel :

Et partout l'azur. L'azur au-dessus. L'azur à droite et à gauche. L'azur devant et par-derrière, mais dessous, c'est la terre, une clairière dans un bois, et dedans, un couple, baisant, un mâle, une femelle, deux humains. L'azur et le coït. $(O C, 10)$

Il porte un nom riche de sens, qui souligne pourtant sa duplicité : Jérusalem. Entre le terrestre et le céleste ${ }^{8}$, le temporel et le spirituel, il fait le pont entre deux univers qui se touchent mais qui ne sont pas superposables. Conscience sans chair, le drone est un exilé qui connaît les sensations, mais pas de façon empirique. De ce fait, il peut mesurer lui-même la distance qui le sépare des corps et des plaisirs charnels :

Les baisers m'exilent et m'excluent. [...] Reconstituer, modéliser les sensations, ça oui, je peux l'obtenir. [...] Mais enfin, rien d'équivalent à l'humaine action de plaire, de séduire, de contenter l'autre et soi. $(O C, 12)$

L'Opium du ciel, le dernier chapitre sera une sorte de Révélation faite au drone.

${ }^{7}$ On note bien sûr l'écho intertextuel à L'Après-midi d'un faune de Stéphane Mallarmé, publié en 1876, qui relate le monologue bucolique d'un faune. Cette églogue a été mise en musique par Claude Debussy dans son Prélude à l'Après-midi d'un faune entre 1892 et 1894. Le compositeur français était à la recherche du son pur grâce à l'utilisation de timbres musicaux insolites et raffinés, afin de créer de nouvelles significations accessibles seulement à quelques initiés. Dans cette œuvre, Debussy emploie des procédés qui donnent une autre sensation du déroulement du temps en musique : ni pulsation appuyée, ni rythme clairement marqué, des harmonies qui s'éloignent des lois des musiciens classiques et romantiques (cf. R. Leibowitz, Le Compositeur et son double, Paris, Gallimard, 1986, p. 252).

8 Psaume 122, 3 : Jérusalem est décrite comme une ville dont les parties sont liées ensemble. Les exégètes ont interprété cette phrase comme une double nature de la cité : à la fois la Jérusalem d'en bas, et la Jérusalem d'en haut. Par analogie, le drone prend vie dans un espace et un temps inscrits dans ceux des humains, mais sa durée de vie illimitée le rend divin. 
Cette privation le réduit à l'isolement et à vivre en marge de la société des hommes. Mû par la solitude, le drone cherche un expédient à ce manque. Pour pis-aller, il entreprend de s'inventer une enveloppe physique par le biais des réseaux sociaux. À l'ère numérique, un jeu shakespearien se déploie grâce aux profils, ces identités fictives où l'on joue à être et à n'être pas. Jérusalem se crée une nouvelle image : il combine tous les atouts pour plaire à la gent féminine mais surtout un " physique de rêve " $(O C, 206)$. Toutefois, cette expérience se conclut par un échec probant. La rencontre virtuelle avec Sarah, décrite comme une mère nourricière et câline, est interrompue brutalement par le drone, conscient du mal qu'il cause à une femme en recherche d'une relation extraconjugale. Une autre aventure, avec une Indienne qui porte le nom de Nayana, le conduit à se faire passer pour un jeune cadre londonien, mais les échanges ne pourront, pour des raisons évidentes, jamais être corporels. Jérusalem demeure un être différent, sans mains, ne pouvant ni manger ni boire, et surtout privé de l'accomplissement du désir charnel.

Sa nature exceptionnelle l'a pourtant rendu curieux. Grâce à ses capteurs multisensoriels lui permettant d'être attentif au monde qui l'entoure, son attention peut se porter sur toutes sortes de faits, qu'ils soient ceux du quotidien ou de l'extraordinaire. Sa position surplombante le rend furtif, ce qui lui permet d'observer les vies minuscules au plus près. Présent et absent, le drone est une caisse de résonance de l'agitation du monde :

J'entends toutes les conversations des téléphones portables. Journée détaillée, joies bancales, lassitudes et prises de tête concertent dans mes micros. As-tu bien travaillé à l'école ? As-tu mangé ? Veux-tu que j'aille faire les courses ? T'es-tu occupé des factures ? Nous ne sommes que tous les deux tu sais ce soir. J'ai envie de toi tu sais ? On se mate quoi ? Viens vite, j'ai hâte et tu me manques... $(O C, 20)$

Son vol est comparable à celui des oiseaux. II migre vers de multiples régions du globe, se rendant en Thaï- 
lande ou en Inde pour y découvrir des peuples différents. Durant ce parcours, il constate le chaos général qu'il résume à l'aide de rapprochements incongrus :

On a, l'autre jour, retrouvé des joggeuses dispersées dans des sacs poubelles, leurs membres mélangés. Au même instant, mais ailleurs, une famille à vélo s'est arrêtée cueillir des champignons et la plus petite des fillettes a composé une couronne de fleurs des bois pour sa maman. $(O C, 17)$

Le beau et le laid coexistent dans le monde moderne, mais c'est bien la noirceur qui l'emporte. Tout voyage est déceptif. L'escapade à Venise, lieu topique de l'amour, se change en un périple stérile et ennuyeux. Jérusalem en fait le constat :

Toutes les émotions avaient déjà eu lieu, chaque génération répétait la même gamme, dans un ordre différent au mieux, mais les notes étaient identiques. Le décor planétaire changeait toujours en pire. [...] L'idée de progrès était relative. $(O C, 186)$

Tout semble voué à l'échec car la noirceur des villes est à l'image de l'âme des individus et plus particulièrement des Hommes. La France n'est plus que le pays des idéologies endormies tandis que l'Asie représente le continent du cynisme et des espoirs déçus. Cela fait écho à la théorie du philosophe Jean-Pierre Dupuy au sujet de l'imminence de la catastrophe : "Nous sommes entrés dans l'ère du sursis $"$.

Ici-bas, le drone constate que le monde est plein d'une violence paroxysmique. Autour de lui, il observe avec un certain effroi des passions dégradées, sans idéal. Même l'amour devient un jeu de pouvoir et de force. Le narrateur, voyeur à l'occasion, décrit dans les moindres détails ces jeux érotiques. Mais ces descriptions ne sont pas là pour le ravir. Au contraire, la vision d'une scène obscène dans une prison $(O C, 200-202)^{10}$ le heurte et met fin

\footnotetext{
9 J.-P. Dupuy, La Marque du sacré, Paris, Flammarion, 2010, p. 62.

10 Dans cette scène, un homme est contraint par la force de copuler avec une femme décrite comme une créature androgyne.
} 
à ses aspirations à trouver la beauté du monde : " Je n'ai pas voulu vivre ça seulement !, je n'ai pas voulu vivre seulement ça !, je n'ai pas seulement voulu vivre ça !" (OC, 202). La répétition avec les variations presque imperceptibles de ce cri du cœur souligne à quel point il est nécessaire de se retrancher ailleurs, loin de ce " théâtreabattoir ».

La seule relation possible pourrait être fantasmée. Peut-être avec celle qui le contrôlait lorsqu'il était un drone militaire :

Nos pilotes vivaient au début près de nous comme un cavalier acclimate sa monture à son odorat son toucher sa voix [...]. Jusqu'à présent, il n'y avait eu qu'une relative froideur, car ils n'entraient pas en nous, ils ne prenaient pas place à l'intérieur de nous, ils ne refermaient pas sur eux la trappe qui les voyait s'asseoir dans notre tête comme avec les traditionnels aéronefs. Nous n'avions jamais eu droit, ou quasi jamais, à ces caresses cent fois montrées au cinéma où l'homme promène sa main sur les courbes d'acier chaud de l'appareil avec la même lenteur que s'il s'agissait d'une promenade le long des carnes douces de l'être aimé. $(O C, 44)$

Mais ce désir de rapport charnel avec Euréka ${ }^{11}$, cette Américaine qui le dirigeait à distance dans les opérations d'espionnage au Moyen-Orient, ne peut être satisfait. Le drone ne possède pas encore sa pleine conscience et doit être formé. Il a tout de même ressenti un embryon d'éveil que seule une transformation radicale peut faire advenir.

Métamorphoses : l'amour de la science substitut à une «déontologie douteuse »

Le roman pose la question de l'engagement. Le drone peut-il rester un simple contemplateur contempteur ? Cet œil omniscient est-il en capacité de changer ce monde déshumanisé ? Le lecteur va assister à la métamorphose

$\overline{11}$ Ce nom a été choisi par Jérusalem « à cause de sa vivacité, de son intelligence tactique et de sa sensibilité stratégique qui la faisaient appuyer sur le bouton de tir sans haine et sans hésitation, à la vitesse de l'idée » $(O C, 46)$. 
de Jérusalem grâce à un neurocier ${ }^{12}$. Cette trouvaille technologique lui confère une vie éternelle :

Mon neurocier garantissait une énergie illimitée, un simulacre d'immortalité sous les cieux terriens. Tant que la Terre serait à sa place et à bonne distance du Soleil et qu'aucun astéroïde ne viendrait y percuter, même les désastres d'en bas ne me toucheraient qu'en raison de ma lâcheté à m'en mêler. (OC, 204)

Mais cela le rend aussi plus "humain ". Son ton initialement inquisiteur et réprobateur devient petit à petit plus doux pour porter un message d'espoir à ses dissemblables. Cela occulte progressivement la question du corps au profit de la recherche de la sagesse.

La fabrication du drone montre bien cette évolution. II est la combinaison d'un drone de compagnie, un simple jouet offert à une adolescente française et qui est décrit comme un être de série, lent, à la "déontologie douteuse ", et d'un drone militaire américain, utilisé dans la guerre au Moyen-Orient et disposant d'un système de transcription archaïque. C'est de l'assemblage de "Lovecraft " et de " CSNR108 " que va naître " Jérusalem ".

Ces changements sont le produit du travail de ses géniteurs, un couple de parias habitant dans le désert du Sinaï. Il est recueilli, tel Moïse, à la différence près que lui n'a pas été sauvé des eaux mais qu'il provient du ciel. Dans un campement de fortune réservé aux proscrits de la communauté scientifique, ces chercheurs vont modifier sa structure et sa vision du monde par la même occasion. En effet, Marija Gimbutas et Raphael Patai, les « plus spécialisés du coin en matière de technologie " $(O C, 88)$ vont le doter d'un nouveau code et partager leur savoir avec lui. Jean-Noël Orengo mêle ici le réel et la fiction en utilisant des scientifiques qui ont réellement existé pour exposer leur travail, tombé en désuétude. La première est spécialisée en archéologie et en sciences du langage. Née en Lituanie, pendant l'ère soviétique, Marija Gimbutas a multi-

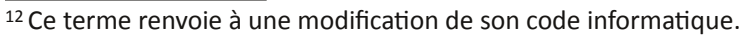


plié les fouilles en Europe et au Moyen-Orient et a découvert l'omniprésence du féminin, sous toutes ses formes. Selon elle, c'est le témoignage laissé pendant près de 25000 ans du culte d'une grande déesse, qui aurait pris fin il y a 6000 ans $^{13}$. Le second est Hongrois et sa judéité le fait s'intéresser à l'histoire de son peuple, avant l'exil à Babylone. La thèse de Raphael Patai met en avant la cohabitation entre Yahvé et Ashérah, une déesse protectrice, nourricière mais surtout détentrice du savoir.

Le travail mené par ses parents suscite autant l'admiration qu'il constitue une source de plaisir pour le drone :

Parfois, leurs voix se chevauchaient, ils continuaient leurs monologues, précisant un point, en amplifiant un autre, et je me taisais, hébété, groggy par leurs caresses argumentatives. (OC, 136)

Cet enseignement est d'autant plus efficace qu'il associe l'amour à donner au savoir à dispenser. Le drone reconnaît l'étrange alchimie de ce mélange qui opère efficacement en lui :

Ils prirent l'un après l'autre la parole assez longuement. II était question de leur vie, de leur rencontre, de leurs travaux, de tout ce qui rend mystérieux des parents passionnants qui ont non seulement de l'amour à donner mais du savoir, des parents d'exception comme nous aimerions tous en avoir et comme nous aimerions tous l'être. $(O C, 104)$

L'être protéiforme, tour à tour simple jouet, arme militaire, outil de surveillance, compagnon de voyage, devient alors un drone unique et pensant, qui va épouser la cause parentale. Cela rejoint la conception freudienne de l'épistémophilie ${ }^{14}$, sublimation de la pulsion sexuelle. Le

13 II s'agit d'une théorie appelée " l'hypothèse kourgane ". Une population vivant au nord de la mer Noire aurait détruit les vieilles sociétés en instaurant la violence. Le judaïsme, avec son dieu unique, jaloux et belliqueux, serait l'héritage de ce peuple.

14 S. Freud, Pulsions et destins des pulsions, trad. Olivier Mannoni, Paris, Petite Bibliothèque Payot, 2018. Le fondateur de la psychanalyse voit en la pulsion sexuelle une énergie qui peut être détournée de son but, et donc utilisée à d'autres fins que sexuelles. II est bien question d'un transfert d'un objet à un autre, et plus particulièrement vers un désir de connaissance. 
désir érotique du drone se transforme en appétence pour la connaissance. Désormais, il est poussé par la libido sciendi et devient un " amoureux scientifiquement » :

Être amoureux scientifiquement n'est pas la même chose qu'être amoureux littérairement, n'est pas la même chose qu'être amoureux sexuellement et sentimentalement de l'être élu, chassé ou condamné par cet amour. Si être amoureux littérairement place l'être aimé sur un piédestal [...], être amoureux scientifiquement consiste à l'allonger sur une table entourée d'instruments, et à l'autopsier sans fin, le mettre en pièces jusqu'à la dernière de ses cellules afin d'en connaître l'ADN [...] et l'anatomie artistiquement justifiée par le bon coup de crayon, de pinceau et de scalpel. $(O C, 144)$

Éveillé par ses parents et d'autres scientifiques ${ }^{15}$ qui participent à son éducation, Jérusalem se considère enfin comme un adulte. II a atteint sa maturité. Son rôle est à présent d'endosser le rôle de guide spirituel. II doit reconstituer la lumière qu'il reçoit pour la répandre dans le monde et devenir " Jérusalem pour tous ". La métaphore insiste sur l'importance de la fraternisation des peuples dans une époque où les conflits font rage. Pour ce faire, il revêt d'abord le costume du témoin :

Être témoin signifiait observer avec bienveillance, user de remarques jolies et très douces, sucrées, comme dans beaucoup de romans français contemporains qui sont d'abord, et avant tout, des bonbonnières. (OC, 173)

Le corps observé dans ses moindres détails disparaît au profit d'une hauteur d'ensemble. Désormais, les silhouettes remplacent les individus, les rencontres se font plus courtes. Jérusalem s'extirpe du monde des vivants et

\footnotetext{
15 II s'agit de Martin Bernal et Jacques Bergier, connus pour des thèses à rebours de la doxa. Le premier a démontré dans Black Athena (M. Bernal, Black Athena: Afroasiatic Roots of Classical Civilization, Volume I: The Fabrication of Ancient Greece, 1785-1985, New Brunswick, NJ, Rutgers University Press, 1987) que les pharaons étaient noirs et qu'ils avaient apporté leur civilisation en colonisant la Grèce. Le second a contribué à la promotion de théories paranormales grâce à son essai intitulé Le Matin des magiciens (J. Bergier, L. Pauwels, Le Matin des magiciens, Paris, Gallimard, 1960) relayées ensuite par la revue Planète. Jérusalem se rêve d’ailleurs en une du magazine au chapitre XI.
} 
la quête change radicalement. C'est à présent une odyssée spirituelle qui se poursuit inlassablement jusqu'à la rencontre fortuite avec l'Arbre. Cet épisode déterminera le rôle final de son existence. C'est l'expérience ésotérique ultime qui façonnera sa destinée. II deviendra mage, " Jérusalem-Merlin ", le prophète qui assiste à la (re)création du monde :

Il y a des astres là-dedans. Des sphères. Aller aux sphères, c'est la seule synagogue. Aller aux sphères écouter la musique d'autres sons continus, sororités sonores [...]. Le nouveau monde, il est béant, humide et chaud. Tout est féminin. $(O C, 264)$

La révélation finale relate l'accouchement d'un monde qui renaît. Le drone, par sa nature suprahumaine, est le porte-parole de ce processus. II est monté vers les cieux pour mieux écouter, pour devenir la caisse de résonance auprès de l'assemblée des humains de cette vibration cosmique qui annihile l'ordre ancien qui est celui de la guerre et des dieux jaloux et possessifs.

Une méditation sur le monde cyclique et privé du féminin

Pour mener à bien sa mission, il est nécessaire pour le drone de se redéfinir, de se reconnaître avant de connaître. Le récit prend alors des accents de recherche de soi avant de poursuivre l'enquête vers l'extérieur. Le parcours de Jérusalem se caractérise dès lors par une fuite vers l'inconnu, un éloignement progressif du monde terrestre vers la découverte du sens profond des choses:

L'objet de la science est de connaître la vérité ; son occupation, de la chercher ; son caractère, de l'aimer ; les moyens de l'acquérir sont de renoncer aux passions, de fuir la dissipation et l'oisiveté. ${ }^{16}$

C'est pourquoi il doit " combler les vides " $(O C, 251)$ en vue d'apporter l'ultime message à l'humanité.

16 J.-J. Rousseau, Esprit, maximes et principes, Neuchâtel, Les libraires associés, 1764 , p. 4. 
Dans la veine rabelaisienne, le voyage du drone est semé d'embûches, de rencontres fortuites et conduit à la découverte d'un oracle qui présente de multiples similitudes avec la dive bouteille ${ }^{17}$. Ces deux odyssées ont pour point commun de révéler le sort de l'humanité entière après avoir multiplié les étapes initiatiques. Elles se concluent sur des visions positives du monde, à des niveaux différents certes, mais qui ont pour caractéristique principale de remettre en mouvement la " branloire pérenne ${ }^{18}$. En effet, lors de leur arrivée à l'oracle de la dive bouteille, Pantagruel et ses acolytes découvrent inscrite sur une table d'airain une sentence tirée du grec : "Toutes choses se meuvent à leur fin ${ }^{19}$. Le dernier chapitre de l'Opium du ciel intitulé "La nuit de Brahma » est une nuit de feu ${ }^{20}$, qui déconstruit toutes les formes d'unité. La syntaxe se disloque, les phrases se heurtent les unes aux autres, la compréhension littérale est mise à mal par une recomposition qui réunit ces éléments en les fusionnant et en les démultipliant de façon exponentielle. Même au niveau minimal, les lettres s'embrasent et se rebrassent pour faire advenir un ordre nouveau :

17 Rabelais, CEuvres complètes, "Cinquième livre ", Paris, Gallimard, 1955. Dans Le Cinquième Livre, publié en 1564, Rabelais relate la suite et fin des aventures de Pantagruel. Le géant, accompagné de ses compagnons, traverse des pays qui sont autant d'épreuves à surmonter. À la différence de celle du drone Jérusalem, cette aventure est collective et mène à une recherche symbolique de la sagesse par la boisson afin d'étancher sa soif et trouver au plus profond de soi la vérité.

18 Montaigne, Essais, III, 2, "Du repentir ", P. Villey, V. L. Saulnier (éd. critique), Paris, P.U.F., 1965, p. 350. Dans cet essai, Montaigne utilise l'expression " branloire pérenne " pour insister sur le mouvement incessant du monde, où rien n'est fixe, où tout est en perpétuel devenir.

${ }^{19}$ Rabelais, CEuvres complètes, "Cinquième livre ", Paris, Gallimard, 1955, p. 859.

20 A. Blanchet, "La nuit de feu de Pascal ", [dans :] Études, novembre 1954, n²83. L'expression " nuit de feu » est une référence à la conversion de Blaise Pascal, telle qu'il l'a relatée dans le Mémorial, daté vraisemblablement du lundi 23 novembre 1654. Ce texte lapidaire et énigmatique relate une expérience mystique qui conduira à son adhésion totale à la spiritualité catholique. Orengo pastiche ce classique en reprenant les tournures ésotériques de l'auteur des Pensées. 
Et l'arbre, c'était qui déjà ?, non plus l'arbre d'un trait, mais le $A$, le $R$, le $B$, le $R$ encore et le $E$, et ainsi délettré puis recomposé peut-on dire indifféremment l'EBRAR, le BRARE, le REBAR, etc., et les cieux se referment, se replient, se froissent, redeviennent une boule dans la main de Brahma qui termine sa journée. $(O C, 263)$

Ce vingt-quatrième chapitre clôt le récit sur une dernière heure sombre qui coïncide avec le retour de la lumière. Le nouveau jour s'ouvre sur une promesse : celle d'un ordre originel rétabli et d'un monde régénéré. Le dieu Brahma est ici convoqué en tant que démiurge qui réincarne toute chose. Jean-Noël Orengo s'appuie en grande partie sur la conception des quatre âges du calendrier hindou et bouddhiste qui fait se succéder au Kali Yuga, "l'âge de fer », celui du Krita Yuga, la période dorée. II la préfère au récit anthropogonique des cinq âges de I'humanité d'Hésiode car l'Inde a gardé une vision du temps avec des cycles qui tournoient indéfiniment.

Ce renouvellement effacera toute trace du passé. Le drone se met ainsi à distance et devient le contemplatif, l'observateur privilégié, le témoin de cette métamorphose :

Mes activités, pour la plupart, étaient méditatives. Chaque expérience anodine, chaque observation banale finissait en formule gravée dans un sillon. [...] J'étais un drone, oui, mais le sens du mot désignant l'espèce à laquelle j'appartenais dépassait ce corps technique. Drone signifie en musique le son continu. Et le son continu garantit les dérives les plus gratuites. Quitter le faux bruit, les médisances pour un truc formel beau comme un son qui ne s'arrête jamais. L'immortalité harmonique. Ça me plaisait bien. La perspective me plaisait bien. Les perspectives des lignes de partition d'un son continu me plaisaient bien. (OC, 224)

Jérusalem finit par rentrer complètement en lui. II ne s'agit plus de vouloir changer les hommes ni même de vouloir les comprendre. II s'agit d'attendre, de laisser le temps s'écouler pour retrouver le temps béni des origines. Le drone finit par s'immobiliser, ce qui se traduit par l'extinction progressive de tout ce qui causait le mouvement et en particulier ses instincts. À l'inverse de l'extase, seule subsiste sa conscience en état $d^{\prime}$ " instase ", pour re- 
prendre le néologisme de Mircea Eliade ${ }^{21}$. Le drone quitte le monde des apparences, un monde facile, léger et bruyant.

\section{Mythes et réalités : la séduction du Verbe}

Le roman explore les possibilités du langage dans toutes ses dimensions. Le drone multiplie les tentatives pour trouver le mot juste, celui qui servira à retranscrire la métamorphose finale :

Il fallait que je revienne sans cesse sur la grammaire de mon identité, cette suite de propositions qui, tout autant que nos cellules, déterminent ce que nous sommes. (OC, 198)

À un niveau supérieur, le récit cherche également sa voie entre genres voisins. II brouille les codes en empruntant au conte ses faits extraordinaires et aux dystopies modernes leur angoisse sur les dérives de notre monde. II reprend à son compte des mythes fondateurs en les intégrant dans notre univers contemporain. L'idylle amoureuse entre Ashérah, la fille biologique de ses concepteurs, et David, un Français, est une reprise de la Genèse. La scène se déroule dans le Morvan qui est décrit comme un havre de paix, comparable au jardin d'Éden. Ses occupants deviennent des Adam et Ève modernes. Mais, ils vont être chassés de ce paradis terrestre par un couple vipérin de tentateurs, Giacomo (qui est l'archétype du libertin puisqu'il porte le même prénom que Casanova) et Louise, avec qui un adultère sera commis.

Le texte reprend également l'univers fantastique de Lovecraft en évoquant le conflit actuel au Moyen-Orient. Le chapitre VI est une description horrifique du fonctionnement de l'État islamique qu'il associe au Necronomi$\operatorname{con}^{22}$, l'ouvrage fictif du mythe de Cthulhu écrit par le

\footnotetext{
21 M. Eliade, Patanjali et le yoga, Paris, Seuil, 1962, p. 80.

22 Le Necronomicon est évoqué dans la nouvelle La Cité sans nom, écrite en 1921 par Howard Phillips Lovecraft. Son auteur fictionnel, Abdul alHazred, surnommé "l'Arabe fou », est assimilé par Orengo à Abou Bakr al-Baghdadi, le calife autoproclamé de DAESH.
} 
romancier américain. Par une vision de l'intérieur, le drone peut exposer les dangers de l'idéologie mortifère qui a cours dans le califat. Le malheur de nos sociétés, ivres de passions identitaires, se lit donc au travers du prisme de l'intertextualité. Ces reflets déformés nous en apprennent plus sur notre monde.

Jean-Noël Orengo s'ingénie donc à actualiser des récits patrimoniaux. Ceux-ci sont une source de connaissance qui séduit l'auteur qui joue avec les codes, brouille les frontières génériques en faisant porter la charpente narratologique à une machine pensante. À nul autre pareil, en mettant de façon unique un drone en guise d'instance énonciatrice, son roman est aussi un objet de séduction. Il oscille sans cesse entre la vulgarisation de savoirs savants et le désir de les ancrer dans la réalité la plus immédiate.

Le ciment de cet édifice est le Verbe de cette créature technicisée. Par sa langue ciselée, virevoltante, semblable au vol de l'albatros baudelairien, le drone est dans un entre-deux : il est pris entre l'azur de beauté et sa gaucherie terrestre. II nous éclaire pourtant en nous invitant à (re)découvrir notre époque en tissant le lien entre passé et présent. L'Opium du ciel est un récit sapiosexuel en ce qu'il manifeste un désir irrépressible de dire en mots et en sons toute la complexité du monde moderne, décrit comme ultrasexué et violent. 


\section{bibliographie}

Bergier J., Pauwels L., Le Matin des magiciens, Paris, Gallimard, 1960.

Bernal M., Black Athena: Afroasiatic Roots of Classical Civilization, Volume I: The Fabrication of Ancient Greece, 1785-1985, New Brunswick, NJ, Rutgers University Press, 1987.

Blanchet A., " La nuit de feu de Pascal ", [dans :] Études, novembre 1954, $\mathrm{n}^{\circ} 283$.

De Mulder C., Libido sciendi ; le savant, le désir, la femme, Paris, Seuil, 2012.

Dupuy J.-P., La Marque du sacré, Paris, Flammarion, 2010.

Eliade M., Patanjali et le yoga, Paris, Seuil, 1962.

Flaubert G., Correspondance, vol. II (juillet 1851 - décembre 1858), J. Bruneau (éd. critique), Paris, Gallimard, 1980.

Freud S., Pulsions et destins des pulsions, trad. Olivier Mannoni, Paris, Petite Bibliothèque Payot, 2018.

Leibowitz R., Le Compositeur et son double, Paris, Gallimard, 1986.

Montaigne, Essais, III, 2, "Du repentir », P. Villey, V. L. Saulnier (éd. critique), Paris, P.U.F., 1965.

Orengo J.-N., L'Opium du ciel, Paris, Grasset, 2017.

Rabelais, CEuvres complètes, "Cinquième livre », Paris, Gallimard, 1955.

Rousseau J.-J., Esprit, maximes et principes, Neuchâtel, Les libraires associés, 1764.

Stone M., Quand dieu était une femme, C. Germain et al. (trad.), MontréalParis, L'Étincelle, 1979.

\section{abstract}

\section{L'Opium du ciel by Jean-Noël Orengo : the spiritual quest of a drone, between asceticism of carnal pleasures and exhibition of bodies}

In his novel intitled L'Opium du ciel (2017), Jean-Noël Orengo narrates the adventures of a special drone owning a conscience. This steel creature will suffer some modifications which will revise his forner identity. The libidinous machine becomes a spiritual entity. Hereafter, he aims to find the meaning of Life. He understands that the catastrophe is imminent because of the depravation of all values. Nevertheless, there is still hope : the cycle of metamorphoses will erase all. The drone will be the prophet of this renascence. The old world and its violence related to sexual desire will be cleared in favor of a more harmonious Earth. 


\section{keywords}

Orengo, Genesis, drone, desire, knowledge

\section{mots-clés}

Orengo, Genèse, drone, désir, savoir

\section{thomas diette}

Thomas Diette est un enseignant agrégé à l'université de Lille (France) et fait partie du laboratoire Alithila. II rédige actuellement une thèse sur les enjeux risqués de la représentation des croyances religieuses dans la littérature contemporaine. En s'intéressant à des auteurs très variés, parmi lesquels Houellebecq, Michon et Carrère, son projet est de mener une réflexion pluridisciplinaire sur la place des religions à une époque où elles reviennent en force dans le champ littéraire, mais aussi dans la société.

ORCID : 0000-0002-7090-2179 